\title{
Discrimination of the commercial Korean native chicken population using microsatellite markers
}

\author{
Nu Ri Choi ${ }^{1}$, Dong Won Seo ${ }^{1}$, Slim Ben Jemaa ${ }^{1,2}$, Hasina Sultana ${ }^{1}$, Kang Nyeong Heo ${ }^{3}$, Cheorun Jo ${ }^{4}$ and Jun Heon Lee
}

\begin{abstract}
Background: Korean native chicken (KNC) is a well-known breed due to its superior meat taste. This breed, however, owing to a low growth rate, has a high market price. In order to overcome this disadvantage, the National Institute of Animal Science (NIAS) in Korea developed a commercial KNC breed, named Woorimatdag version 2 (WM2), an upgraded version of the Woorimatdag (WM1) breed and the WM2 was created by crossing the KNC with meat type breeds. This study aims to discriminate between WM2 and other chicken breeds using microsatellite (MS) markers.

Methods: A total of 302 individuals from eight Korean chicken populations were examined. The genetic diversity and population structure analysis were investigated using Cervus, API-CALC, STRUCTURE, PowerMarker programs.

Results: Based on heterozygosity and polymorphic information content (PIC) values, 30 MS markers were initially selected from 150 markers. The identified average number of alleles ( $\mathrm{Na}$ ), expected heterozygosity, and PIC values for the WM2 samples were $7.17,0.741$, and 0.682 , respectively. Additionally, the paternity of individuals was assigned with a success rate of greater than $99 \%$ using 12 markers, the best minimum number of markers. The 12 selected markers contained heterozygosity and PIC values above 0.7 and probability of identity values around zero. Using these markers, the determined probability of identity $(P I)$, $P l_{\text {half-sibs, }}$ and $P I_{\text {sibs }}$ values were $3.23 \mathrm{E}-33,5.03 \mathrm{E}-22$, and 8.61E-08, respectively.

Conclusions: WM2 is well differentiated with respect to other chicken breeds based on estimated genetic distances. The results presented here will contribute to the identification of commercial WM2 chicken in the market.
\end{abstract}

Keywords: Discrimination, Diversity, Microsatellite, Korean native chicken, Woorimatdag

\section{Background}

Recently, chicken meat consumption in Korea has rapidly increased to $12 \mathrm{~kg}$ per capita due to consumer preferences for healthy white muscle meat [1]. In comparison to red meat, chicken meat is considered a healthier option because of lower fat, cholesterol, and iron levels [2]. Presently, approximately $90 \%$ of the Korean poultry industry contains imported chicken breeding stocks. The breeds that existed before the Korean War (1950-1953), unfortunately, are almost all extinct. Since 1992, a Korean native chicken (KNC) conservation project was launched by the National Institute of Animal Science (NIAS) in an attempt to restore local chicken breeds. Recently, five KNC lines and seven others originally imported in the 1960s have been restored [3]. Consumers tend to pay more for the KNCs because of

\footnotetext{
* Correspondence: junheon@cnu.ac.kr

'Department of Animal Science and Biotechnology, College of Agriculture and Life Sciences, Chungnam National University, Daejeon 305-764, Republic of Korea

Full list of author information is available at the end of the article
}

their good taste. The low productivity of the native breeds, however, was disadvantageous for farmers trying to meet feeding and consumption rates. In order to overcome these disadvantages, NIAS developed the Woorimatdag version 1 (WM1) chicken population. WM1 was a commercial, KNC population generated from crossbreeding fast growing native male chickens and good tasting female chickens with increased egg production. WM1 chicken grows faster, reaching the marker weight of $1.8 \mathrm{~kg}$, than the purebred KNC [4]. Moreover, WM1 chickens produce good quality meat with a high oleic acid content, which improves both taste and water holding capacity [4]. Jung et al. [5] reported that WM1 chickens have a significantly higher content of arachidonic acid and meat flavor than commercial broilers (Br). NIAS recently developed Woorimatdag version 2 (WM2) chickens, a modified version of WM1 chickens with increased growth rates.

Traditional methods to identify chicken breeds focused on general appearances such as feather color, shank color, 
and body type [6]. Because of the process of distribution and market, chicken breeds cannot be effectively classified based on the appearance of meat. Recent advances in molecular biology techniques, however, have provided new opportunities to assess genetic variability at the DNA level [7]. Therefore, many groups have attempted to discriminate breeds using molecular genetic markers. For example, Korean cattle and pork industries developed discrimination and traceability systems using microsatellite (MS) markers [8,9].

MS markers or simple-sequence repeat (SSR) markers, are highly polymorphic, one to six base pair repeats, widely used since they are numerous, randomly distributed in the genome, and show co-dominant inheritance $[10,11]$. In addition, MS markers were used in the construction of linkage map of quantitative trait locus (QTL) studies [12]. MS markers may be useful in discriminating individuals. The International Society for Animal Genetics (ISAG) has recommended 30 MS markers for breed identification [13].

In a previous study, $97 \mathrm{MS}$ marker variations, including the 30 MS ISAG recommended markers were investigated in 12 chicken populations. While the majority of the 12 population studies were purebred, the commercial chickens were different, products of three- and four-way crosses. Therefore, in this study, the commercial KNC population, WM2 was investigated to discriminate it from the other chicken populations in the market.

\section{Methods}

\section{Sample collection and DNA extraction}

A total of 302 individuals from eight Korean chicken population (187 WM2, 17 WM1, 13 Hanhyup-3 (Hh), 14 Hyunin (Hn), 14 Rhode Island Red (RIR), 15 Cornish Black (CoL), 15 Cornish Red (CoR), 17 Ogye (O) and 10 $\mathrm{Br})$ were examined. Chicken populations care facilities and procedures met or exceeded the standards established by the Committee for Accreditation of Laboratory Animal Care at National Institute of Animal Science (NIAS) in Korea. The study also was conducted in accordance with recommendations described in "The Guide for the Care and Use of Laboratory Animals" published by the institutional Animal Care and Use Committee (IACUC) of NIAS (2012-C-037) in Korea. Genomic DNA was extracted from embryo tissues of WM2 using the PrimePrep ${ }^{\mathrm{Tm}}$ Genomic DNA isolation kit for tissue (GeNetBio, Korea) and blood samples of other population using the PrimePrem ${ }^{\text {Tw }}$ Genomic DNA isolation kit for blood. The concentration of DNA samples was measured using NanoDrop 2000C spectrophotometer (Thermo Scientific, USA) and stored at $-20^{\circ} \mathrm{C}$.

\section{Microsatellite (MS) marker genotyping}

Previously, 150 MS markers were investigated for the discrimination of five purebred KNC lines [14]. From these results, a total of 30 MS markers were initially selected, which have high expected heterozygosity (Hexp) and polymorphic information content (PIC) values for classification of the WM2 and other commercial populations (Table 1). Selected 30 MS markers were distributed on 15 autosomes.

The Polymerase Chain Reaction (PCR) was performed in total volume of $20 \mu \mathrm{L}, 50 \mathrm{ng}$ of genomic DNA, 10 pmol of fluorescent dye (FAM, VIC, NED, PET) labeled modified forward primer and normal reverse primer (Applied Biosystems, USA), $2.5 \mathrm{mM}$ of each dNTPs (GeNet Bio, Korea), $10 \mathrm{X}$ reaction buffer (GeNet Bio, Korea), 2.5 unit of prime Taq DNA polymerase (GeNet Bio, Korea). The PCR was performed in an initial denaturation at $95^{\circ} \mathrm{C}$ for $10 \mathrm{~min}$ followed by 35 cycles of $30 \mathrm{sec}$ of denaturation at $95^{\circ} \mathrm{C}, 30 \mathrm{sec}$ of annealing at $60^{\circ} \mathrm{C}, 30 \mathrm{sec}$ of extension at $72^{\circ} \mathrm{C}$ and final extension at $72^{\circ} \mathrm{C}$ for $10 \mathrm{~min}$ using My-Genie 96 Thermal Cycler (Bioneer, Korea). The PCR products were initially electrophoresis on 3\% agarose gel with ethidium bromide $(\mathrm{EtBr})$ and confirmed whether they gave single PCR DNA band under the UV light. When the bands were clearly appeared, further genotyping was performed. For the microsatellite genotyping, more than 20 times diluted PCR products were used. The genotyping reaction contained $1 \mu \mathrm{L}$ of diluted PCR products, $10 \mu \mathrm{L}$ of $\mathrm{Hi}-\mathrm{Di}^{\mathrm{m}}{ }^{\mathrm{m}}$ Formamide (Applied Biosystems, USA) and $0.1 \mu \mathrm{L}$ of GeneScan $^{\mathrm{m}}-500 \mathrm{LIZ}^{\mathrm{mm}}$ size standard marker (Applied Biosystems, USA). After dilution, genotyping reaction mixture was denatured for $2 \mathrm{~min}$ at $95^{\circ} \mathrm{C}$ and fragment analysis was performed using capillary array in Genetic analyzer 3130xl (Applied Biosystems, USA). The MS genotypes were identified using GeneMapper ver.3.7 (Applied Biosystems, USA).

\section{Genetic diversity and population structure analysis}

The genotyping data were used to estimate mean number of allele $(\mathrm{Na})$, Hexp, observed heterozygosity (Hobs) and PIC using Cervus 3.0 program [15]. The expected probability of identity values among genotypes of random individuals (PI), random half sibs $\left(\mathrm{PI}_{\text {half-sibs }}\right)$ and random sibs $\left(\mathrm{PI}_{\text {sibs }}\right.$ ) were calculated using API-CALC (Average Probability of Identity-Calculate) ver 1.0 [16]. Moreover, we used both model-based and non model-based methods to describe the diversity between pre-defined genetic clusters. We used STRUCTURE software v. 2.3 [17] for model-based and DAPC (Discriminant Analysis of Principal Components) program implemented in adegenet R-package [18-20] for non model-based method. STRUCTURE results were also used to assess population structure of Korean breeds.

DAPC analysis was preceded by the execution of the $K$-means clustering algorithm implemented in adegenet to identify an optimal number of genetic clusters to describe the data. For this purpose, we ran $K$-means 
Table 1 Primer information for $\mathbf{3 0}$ microsatellite markers used in this study*

\begin{tabular}{|c|c|c|c|c|c|}
\hline Marker & Chr. & Dye & Forward $\left(5^{\prime} \rightarrow 3^{\prime}\right)$ & Reverse $\left(5^{\prime} \rightarrow 3^{\prime}\right)$ & Allele size (bp) \\
\hline ADL0268 & 1 & PET & СTCCACCCCTCTCAGAACTA & CAACTTCCCATCTACCTACT & $105-117$ \\
\hline MCW0111 & 1 & NED & GCTCCATGTGAAGTGGTTAA & ATGTCCACTTGTCAATGATG & $98-112$ \\
\hline MCW0145 & 1 & FAM & ACTTTATTCTCCAAATTTGGCT & AAACACAATGGCAACGGAAAC & $181-211$ \\
\hline MCW0063 & 2 & FAM & GGCTCCAAAAGCTTGTTCTTAGCT & GAAAACCAGTAAAGCTTCTTAC & $132-150$ \\
\hline MCW0087 & 2 & NED & ATTTCTGCAGCCAACTTGGAG & CTCAGGCAGTTCTCAAGAACA & $267-283$ \\
\hline LEI0141 & 2 & FAM & CGCATTTGATGCATAACACATG & AAGGCAAACTCAGCTGGAACG & $220-242$ \\
\hline MCW0039 & 2 & VIC & CATTGGACTGAGATGTCACTGCAG & ACATTTGTCTAATGGTACTGTTAC & $127-147$ \\
\hline MCW0264 & 2 & FAM & CTTACTTTTCACGACAGAAGC & AGACTGAGTCACACTCGTAAG & $224-240$ \\
\hline MCW0288 & 2 & FAM & GATCTGCTTCTCTGCCCCATG & GGTACTGTCACCAGAATGAGC & $108-122$ \\
\hline MCW0127 & 3 & $\mathrm{VIC}$ & GAGTTCAGCAGGAATGGGATG & TGCAATAAGAGAAGGTAAGGTC & $227-241$ \\
\hline MCW0040 & 3 & $\mathrm{VIC}$ & ACTCAAAAATGTGGTAGAATATAG & ACCGAAATTGAGCAGAAGTTA & $121-145$ \\
\hline ADL0317 & 4 & FAM & AGTTGGTTTCAGCCATCCAT & CCCAGAGCACACTGTCACTG & 178-204 \\
\hline LEI0094 & 4 & FAM & GATCTCACCAGTATGAGCTGC & TCTCACACTGTAACACAGTGC & $254-280$ \\
\hline ADL0292 & 5 & FAM & CCAAATCAGGCAAAACTTCT & AAATGGCCTAAGGATGAGGA & 110-138 \\
\hline MCW0029 & 5 & $\mathrm{VIC}$ & GTGGACACCCATTTGTACCCTATG & CATGCAATTCAGGACCGTGCA & 139-189 \\
\hline ROS0013 & 5 & NED & TGCTGCTCCTGGRAAATTG & GAAAAGCCATGGAGGAATCA & $220-242$ \\
\hline ADL0159 & 6 & VIC & GCCATTATTTTTCCCTGTGT & CTCCCCAAAGTCATTAGCAG & $107-127$ \\
\hline ROS0019 & 7 & NED & ATGTACAGGTTCCAGTGTCCG & CCAGTTCATACAACCTTGAGTTGG & 119-143 \\
\hline ADL0259 & 9 & $\mathrm{VIC}$ & СTCATTGCAGAGGAAGTTCT & GTAATGGAGGATGCTCAGGT & $107-129$ \\
\hline GCT0016 & 9 & NED & TCCAAGGTTCTCCAGTTC & GGCATAAGGATAGCAACAG & $109-125$ \\
\hline MCW0228 & 10 & PET & GATCTCTGCATTACAAGCATG & TTGCTGACCTGCTCATGCAAG & $221-239$ \\
\hline MCW0104 & 13 & FAM & TAGCACAACTCAAGCTGTGAG & AGACTTGCACAGCTGTGTACC & $189-225$ \\
\hline ROS0083 & 13 & $\mathrm{VIC}$ & CATTACAGCTCAGTGTTGGCA & TTGCAAGTGCTCTCCCATC & $109-129$ \\
\hline MCW0213 & 13 & NED & GACAAGTCAACAACTTGCCAG & CTGTTCACTTTAAGGACATGG & 288-316 \\
\hline MCW0123 & 14 & FAM & CCACTAGAAAAGAACATCCTC & GGCTGATGTAAGAAGGGATGA & $79-89$ \\
\hline ADL0293 & 17 & PET & GTAATCTAGAAACCCCATCT & ACATACCGCAGTCTTTGTTC & $105-119$ \\
\hline MCW0330 & 17 & $\mathrm{VIC}$ & TGGACCTCATCAGTCTGACAG & AATGTTCTCATAGAGTTCCTGC & $254-286$ \\
\hline ADL0304 & 18 & FAM & GGGGAGGAACTCTGGAAATG & CCTCATGCTTCGTGCTIIIT & $137-159$ \\
\hline LEI0074 & 26 & VIC & GACCTGGTCCTGACATGGGTG & GTTTGCTGATTAGCCATCGCG & $224-240$ \\
\hline LEI0135 & 28 & NED & CACAATGAAGGATGAATAGTGC & AATTCACAGTTACACCTGAGG & $131-142$ \\
\hline
\end{tabular}

*Bold is selected 12 MS marker combination.

sequentially with increasing number of clusters. Different clustering solutions are compared using Bayesian Information Criterion (BIC). The optimal number of groups matches the lowest BIC value. Also, genetic distance [21] values were computed by PowerMarker ver 3.25 [22]. We used the number of loci which differ between two individuals as a measure of the genetic distance between individuals. This was computed using the $\mathrm{R}$ package ape [23]. A neighbor-joining tree was then constructed based on the resulting distance matrix using the same package. Genetic distance between breeds was computed using Reynolds genetic distance (which is an allele frequency-dependent distance). NeighborNet graph was computed using splitstree software [24].

\section{Results and discussion}

Polymorphisms of microsatellite markers

A total of 215 alleles were detected from 30 MS markers in WM2, ranging from 5 to 13 alleles with an average of 7.17 alleles per locus. The Hexp values ranged from 0.474 for ADL0304 to 0.841 for MCW0264. The Hobs values varied from 0.151 for GCT0016 to 0.885 for ADL0159, with an average of 0.741 per locus in the WM2 population. The obtained average PIC value per locus in WM2 was 0.682 and varied from 0.443 for ADL0304 to 0.819 for MCW0264 (Table 2). These markers were also polymorphic in other chicken populations. The lowest value of $\mathrm{Na}, \mathrm{Hexp}$, and PIC was calculated in the RIR population (Additional file 1: Table S1). Seo et al. [14] 
Table 2 The heterozygosity (Hobs and Hexp) and polymorphism information content (PIC) values using 30 MS markers in Woorimatdag version 2 (WM2) chicken population*

\begin{tabular}{|c|c|c|c|c|}
\hline Locus & No. of allele & HObs & HExp & PIC \\
\hline ADL0268 & 5 & 0.783 & 0.742 & 0.703 \\
\hline MCW0111 & 5 & 0.789 & 0.695 & 0.654 \\
\hline MCW0145 & 7 & 0.754 & 0.692 & 0.655 \\
\hline MCW0063 & 7 & 0.775 & 0.783 & 0.746 \\
\hline MCW0087 & 9 & 0.833 & 0.785 & 0.751 \\
\hline LEI0141 & 7 & 0.832 & 0.797 & 0.767 \\
\hline MCW0039 & 7 & 0.738 & 0.765 & 0.726 \\
\hline MCW0264 & 8 & 0.877 & 0.841 & 0.819 \\
\hline MCW0288 & 6 & 0.608 & 0.631 & 0.578 \\
\hline MCW0127 & 6 & 0.742 & 0.679 & 0.627 \\
\hline MCW0040 & 6 & 0.813 & 0.695 & 0.656 \\
\hline ADL0317 & 7 & 0.819 & 0.775 & 0.739 \\
\hline LEI0094 & 7 & 0.845 & 0.716 & 0.665 \\
\hline ADL0292 & 7 & 0.774 & 0.733 & 0.684 \\
\hline MCW0029 & 13 & 0.805 & 0.815 & 0.790 \\
\hline ROS0013 & 8 & 0.871 & 0.771 & 0.734 \\
\hline ADL0159 & 7 & 0.885 & 0.789 & 0.757 \\
\hline ROS0019 & 8 & 0.766 & 0.643 & 0.581 \\
\hline ADL0259 & 6 & 0.674 & 0.657 & 0.620 \\
\hline GCT0016 & 9 & 0.151 & 0.804 & 0.773 \\
\hline MCW0228 & 6 & 0.821 & 0.773 & 0.740 \\
\hline MCW0104 & 11 & 0.743 & 0.813 & 0.786 \\
\hline ROS0083 & 8 & 0.818 & 0.749 & 0.707 \\
\hline MCW0213 & 11 & 0.807 & 0.798 & 0.771 \\
\hline MCW0123 & 5 & 0.707 & 0.687 & 0.627 \\
\hline ADL0293 & 7 & 0.701 & 0.554 & 0.522 \\
\hline MCW0330 & 6 & 0.484 & 0.643 & 0.592 \\
\hline ADL0304 & 5 & 0.513 & 0.474 & 0.443 \\
\hline LEI0074 & 6 & 0.85 & 0.745 & 0.704 \\
\hline LEI0135 & 5 & 0.659 & 0.596 & 0.544 \\
\hline Mean & 7.17 & 0.741 & 0.721 & 0.682 \\
\hline
\end{tabular}

*Bold is selected 12 MS marker combination.

reported the classification of five different lines of KNC using $15 \mathrm{MS}$ markers and determined the mean $\mathrm{Na}$, Hexp, Hobs, and PIC values of 8.4, 0.802, 0.709, and 0.771 , respectively. With the exception of the Hobs values, the values in the current study were lower than those reported by Seo et al. [14]. Of the 30 selected markers, six (GCT0016, MCW0029, MCW0063, MCW 0087, MCW0264, and MCW0104) were used in the study by Seo et al. [14]. Furthermore, four markers (LEI0135, MCW0111, MCW0145, and MCW0330) were investigated by Suh et al. [25] for the discrimination of four different breeds, including the WM1 population. Our marker combination is more polymorphic than that in the study by Suh et al. [25]. In addition, of the 30 markers in this study, six were among the ISAG recommended markers (ADL0268, LEI0094, MCW0104, MCW0111, MCW0123, and MCW0330). These results indicated that highly polymorphic MS markers were commonly used in diverse populations.

Bostein et al. [26] reported that markers with PIC values $>0.5$ and Hexp values $>0.6$ have a high polymorphic information content and are sufficient for breed discrimination. Thus, our results confirmed that all markers, with the exception of ADL0304, have high polymorphic information and suitable allele frequencies and polymorphisms, and can be used to discriminate the WM2 population.

\section{Discrimination of WM2 population}

While all 30 selected markers had the ability to discriminate the WM2 population from other populations, the best minimum number of markers was required from an economic point of view. For this reason, 12 MS markers were selected for the best minimum MS marker combination based on the highest Hexp and PIC values. Using this combination of 12 MS markers, the calculated PI, $P I_{\text {half-sibs }}$, and $P I_{\text {sibs }}$ values were $3.23 \mathrm{E}-33,5.03 \mathrm{E}-22$, and $8.61 \mathrm{E}-08$, respectively. A previous study, using 12 markers, reported PI, $P I_{\text {half-sibs, }}$ and $P I_{\text {sibs }}$ values of $7.98 \mathrm{E}-29,2.28 \mathrm{E}-$ 20 , and $1.25 \mathrm{E}-8$, respectively, for the discrimination of 5 lines of purebred KNCs [14]. These results suggest that the selected 12 markers have high polymorphism and are effective in discriminating the WM2 population from other populations (Figure 1).

\section{Genetic distance among WM2 and other populations}

To establish genetic relationships among WM2 and the other populations, genetic distances were calculated using the alleles from the 12 selected MS markers. Nei et al. [21]'s genetic distance was calculated between WM2 and the other populations using a pairwise coancestry matrix according to the allele frequencies (Table 3). The lowest genetic distance (0.1375) was observed between the WM1 and CoL populations. The genetic distance between $\mathrm{O}$ chicken population and WM2 was the highest (0.791), followed by RIR and WM2 (0.788) (Table 3). Similarly, Suh et al. [25] reported the lowest genetic distance (0.092) between the WM1 and Hh populations and the highest genetic distance $(0.690)$ between the RIR and White Leghorn breeds. This indicated that the WM1 and Hh populations originated from the same breed/ancestor for constructing the populations. Furthermore, according to the genetic distance values, our marker combination has a stronger discriminating power than that in the findings by Suh et al. [25]. On the other hand, close genetic distances of the WM1 population with 

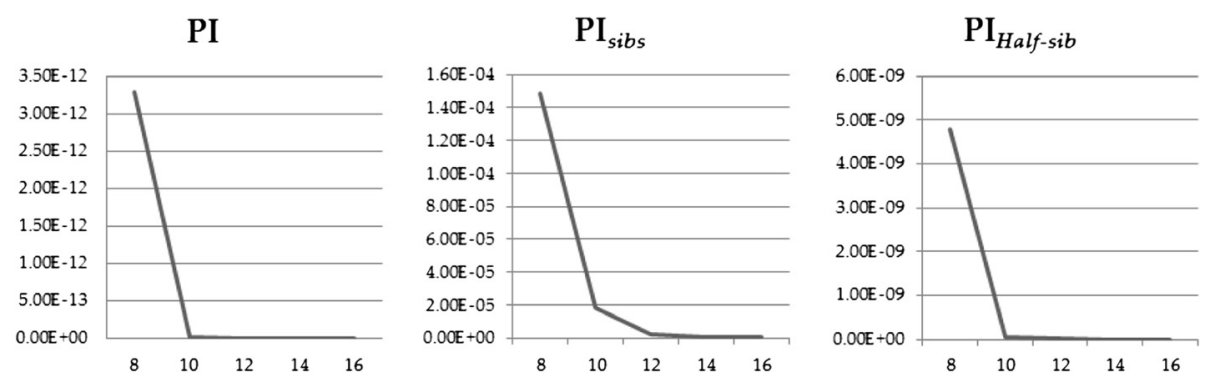

Figure 1 The expected probability of identity values among genotypes of random individuals $(P I)$, random half-sib $\left(P I_{\text {half-sibs }}\right)$ and random sibs $\left(P I_{\text {sibs }}\right)$ were suggested markers for discrimination of chicken lines.

the CoL, Hh, and RIR populations $(0.1375,0.2453$, and 0.2478 , respectively) were observed. These results support the findings by Suh et al. [25] that the WM1 and Hh populations have the same founder breeds as their genetic distances are close (Table 3). The WM2 population, however, has a genetic distance $>0.690$ indicating that different crossing combinations were applied between the WM2 and WM1 populations.

\section{Phylogenetic and structure analysis of nine populations}

Based on Nei's equations [21], an unrooted neighborjoining (NJ) phylogenetic tree was constructed for 263 animals from nine chicken populations using $12 \mathrm{MS}$ marker variations (Figure 2). In our individual phylogenetic analysis, the WM2 population was identified as a distinct population from other populations. O chicken population was also well separated from other populations and a mixture clade contained the WM1, Hh, CoL, and $\mathrm{Br}$ populations.

The genetic structure of nine native chicken populations using microsatellite marker genotypes was investigated based on population clustering (Figure 3). The purpose of structure analysis, performed using a Bayesian approach based on the marker genotypes, was to delineate clusters of individuals [27]. Using 12 MS markers and a K value of 2, WM2 was fully separated from the other populations.
This result was also observed in the individual phylogenetic and discriminant analyses. Based on these results, the combination of 12 MS markers could discriminate WM2 from the other populations. Furthermore, with a $\mathrm{K}$ value of 9, most populations classified well with different groups. WM1, however, was found to be a mixture population, a finding consistent with the results obtained from the phylogenetic and DAPC analyses. The discriminant analysis confirmed the WM2 populations were distinct from the other populations.

The assignment of the individuals from the 9 populations was 9 clusters (group), represent genetic groups and they were inferred using $K$-means algorithm implemented in the R package adegenet (Figure 4). Furthermore, individuals (represented by dots) were plotted according to their coordinates on the first two principal components. The populations were represented as inertia ellipses, which characterize the dispersion of each population around its center of gravity. Bar graph insets indicate the amount of variance determined by the two discriminant values used for plotting. WM2 is clearly separated from the other populations, a result supported by the phylogenetic analysis using 12 selected MS markers. The 12 selected MS markers were also used for the separation of the $\mathrm{O}$ chicken and RIR groups.

Table 3 Matrix of genetic distances among nine populations*

\begin{tabular}{|c|c|c|c|c|c|c|c|c|c|}
\hline & $\mathrm{Bb}$ & CoL & CoR & $\mathrm{Hh}$ & $\mathrm{Hn}$ & 0 & RIR & Wm1 & Wm2 \\
\hline$\overline{\mathrm{Bb}}$ & - & & & & & & & & \\
\hline CoL & 0.3267 & - & & & & & & & \\
\hline CoR & 0.3251 & 0.3906 & - & & & & & & \\
\hline $\mathrm{Hh}$ & 0.2715 & 0.3114 & 0.3805 & - & & & & & \\
\hline $\mathrm{Hn}$ & 0.4189 & 0.4090 & 0.4738 & 0.4460 & - & & & & \\
\hline 0 & 0.4587 & 0.4027 & 0.4543 & 0.4972 & 0.5287 & - & & & \\
\hline RIR & 0.4371 & 0.4702 & 0.5382 & 0.3494 & 0.5492 & 0.6302 & - & & \\
\hline Wm1 & 0.2791 & 0.1375 & 0.3365 & 0.2453 & 0.3667 & 0.4341 & 0.2478 & - & \\
\hline Wm2 & 0.7102 & 0.7225 & 0.7095 & 0.7121 & 0.7618 & 0.7914 & 0.7878 & 0.6904 & - \\
\hline
\end{tabular}

*WM1 (Woorimatdag version 1), WM2 (Woorimatdag version 2), Hh (Hanhyup-3), Hn (Hyunin), RIR (Rhode Island Red), CoL (Cornish black), CoR (Cornish red), $\mathrm{O}$ (Ogye) and $\mathrm{Br}$ (Broiler). 


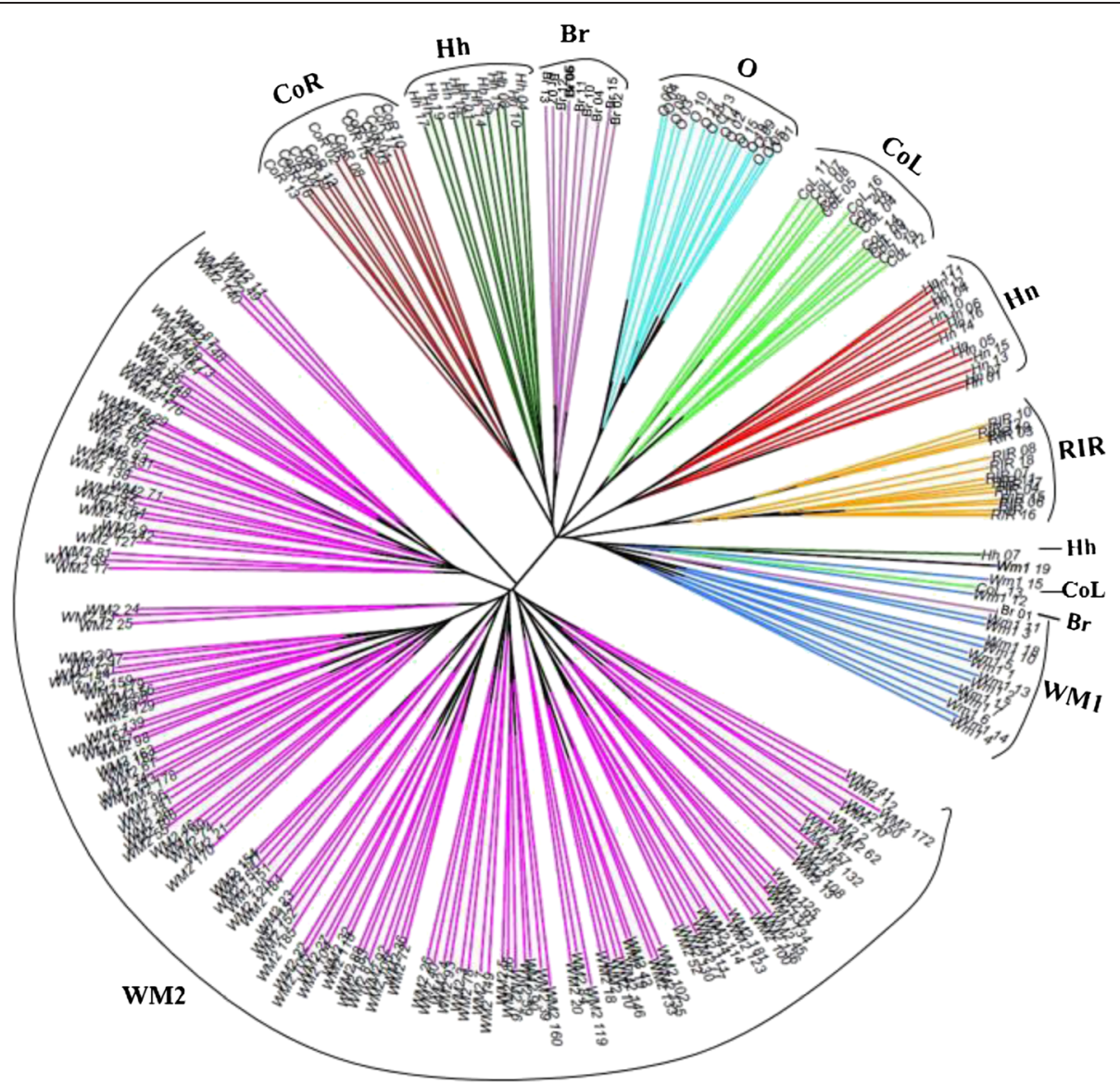

Figure 2 Phylogenetic analysis for each individual from nine populations using Reynolds genetic distance. The color codes are indicated different populations. The population acronyms are as follows: WM1 (Woorimatdag version 1), WM2 (Woorimatdag version 2), Hh (Hanhyup-3), Hn (Hyunin), RIR (Rhode Island Red), CoL (Cornish black), CoR (Cornish red), O (Ogye) and Br (Broiler).

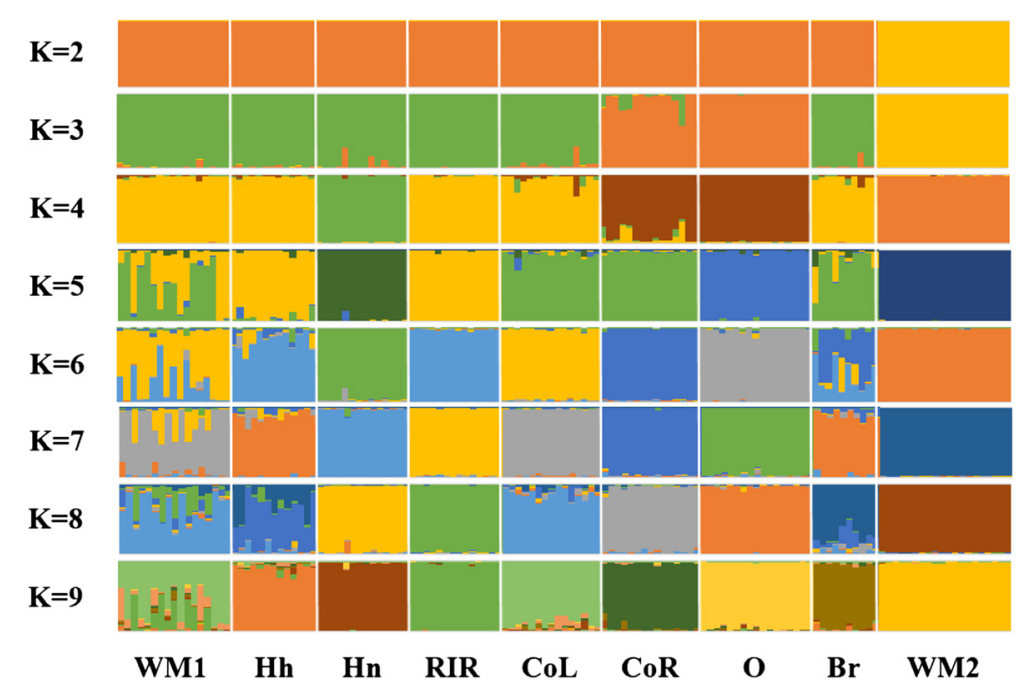

Figure 3 Structure analysis using twelve MS markers from nine populations. The population acronyms are as follows: WM1 (Woorimatdag version 1), WM2 (Woorimatdag version 2), Hh (Hanhyup-3), Hn (Hyunin), RIR (Rhode Island Red), CoL (Cornish black), CoR (Cornish red), O (Ogye) and $\mathrm{Br}$ (Broiler). 


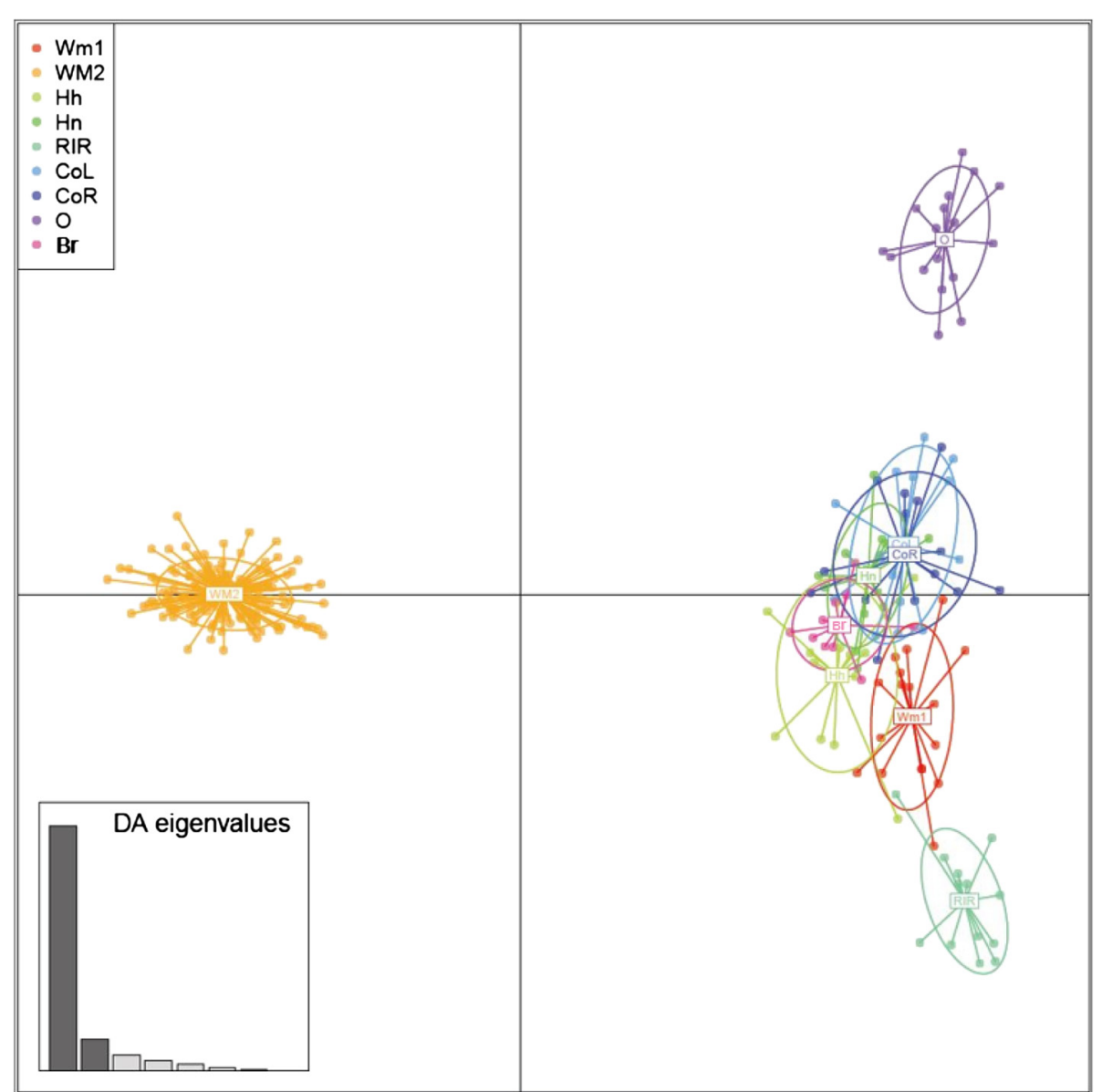

Figure 4 Scatter plot of DAPC analysis of the nine populations using adegent $\mathbf{R}$ package. The population acronyms are as follows: WM1 (Woorimatdag version 1), WM2 (Woorimatdag version 2), Hh (Hanhyup-3), Hn (Hyunin), RIR (Rhode Island Red), CoL (Cornish black), CoR (Cornish red), $\mathrm{O}$ (Ogye) and $\mathrm{Br}$ (Broiler).

\section{Conclusions}

Since our 12 MS marker combination can effectively discriminate WM2, they can be used for breed identification. Moreover, to the best of our knowledge, this is the first study demonstrating the discrimination of the commercial KNC population, and the results presented here may be applied in the commercial market.

\section{Additional file}

Additional file 1: Table S1. The calculated number of alleles ( $k$ ), observed heterozygosity (HObs), expected heterozygosity (HExp), and polymorphic information content (PIC) values in eight chicken populations.

\section{Abbreviations}

BIC: Bayesian Information Criterion; Br: Broiler; CoL: Cornish Black; CoR: Cornish Red; DAPC: Discriminant Analysis of Principal Components; EtBr: Ethidium Bromide; Hexp: Expected heterozygosity; Hh: Hanhyup-3; Hobs: Observed heterozygosity; Hn: Hyunin; ISAG: International Society for Animal Genetics; KNC: Korean native chicken; MS: Microsatellite; Na: Number of allele; NIAS: National Institute of Animal Science; NJ: Neighbor-joining; O: Korean Ogye; PCR: Polymerase Chain Reaction; PI: Probability of identity;
PIC: Polymorphic Information content; QTL: Quantitative Trait Locus; RIR: Rhode Island Red; SSR: Simple-Sequence Repeat; WM1: Woorimatdag version 1; WM2: Woorimatdag verson 2.

\section{Competing interests}

The author declares that they have no competing interests.

\section{Authors' contributions}

NRC, DWS, KNH, CJ and JHL designed experiment, NRC, DWS and HS Carried out lab works, NRC, DWS, SBJ, HS, KNH analyzed data, NRC, DWS, SBJ, CJ, JHL written manuscript. All authors read and approved the final manuscript.

\section{Acknowledgements}

This study was supported by a grant from the Golden Seed Project (No. 2013005042SB730), Korea Institute of Planning \& Evaluation for Technology in Food, Agriculture Forestry \& Fisheries (IPET), Republic of Korea.

\section{Author details}

${ }^{1}$ Department of Animal Science and Biotechnology, College of Agriculture and Life Sciences, Chungnam National University, Daejeon 305-764, Republic of Korea. ${ }^{2}$ INRA-Tunisie, Laboratoire des Productions Animales et Fourragères, Rue Hédi Karray 2049Ariana, Tunisia. ${ }^{3}$ Poultry Science Division, National Institute of Animal Science, RDA, Cheonan 331-801, Republic of Korea. ${ }^{4}$ Department of Agricultural Biotechnology, Center for Food and Bioconvergence, and Research Institute for Agriculture and Life Science, Seoul National University, Seoul 151-921, Republic of Korea. 
Received: 23 December 2014 Accepted: 20 January 2015

Published online: 05 February 2015

\section{References}

1. Ministry of Agriculture, Food and Rural Affairs. Primary statistics of Ministry of Agriculture, Food and Rural Affairs. 2014, Korea

2. Jaturasitha S, Srikanchai T, Kreuzer M, Wicke M. Differences in carcass and meat characteristics between chicken indigenous to northern Thailand (Black-boned and Thai native) and imported extensive breeds (Bresse and Rhode Island Red). Poult Sci. 2008;87:160-9.

3. Heo KN, Choo HJ, Seo BY, Park MN, Jung KC, Hwangbo J, et al. Investigation of TYR and MC1R polymorphisms in Korean native chickens and the commercial chickens. CNU J Agric Sci. 2011;38:465-71.

4. Park MN, Hong EC, Kang BS, Kim HK, Kim JH, Na SH, et al. Chemical composition and meat quality of crossbred Korean native chickens (KNC). Korean J Poult Sci. 2010;37:415-21.

5. Jung YK, Jeon HJ, Jung S, Choe JH, Lee HJ, Heo KN, et al. Comparison of quality traits of thigh meat from Korean native chickens and broilers. Korean J Food Sci Ani resour. 2011;31:684-92.

6. Park MN, Kim TH, Lee HJ, Choi JA, Heo KN, Kim CD, et al. Genetic variatiion of chicken MC1R gene and associations with feather color of Korean native chicken (KNC) 'Woorimatdag'. Korean J Poult Sci. 2013:40:139-45.

7. Hillel J, Groenen MA, Tixier-Boichard M, Korol AB, David L, Kirzhner VM, et al. Biodiversity of 52 chicken populations assessed by microsatellite typing of DNA pools. Genet Sel Evo. 2003;35:533-58.

8. Lim HT, Seo BY, Jung EJ, Yoo CK, Yoon DH, Jeon JT. A comparison of discriminating powers between 14 microsatellite markers and 60 SNP markers applicable to the cattle identification test. J Anim Sci Technol. 2009;51:353-60.

9. Lim HT, Seo BY, Jung EJ, Yoo CK, Zhong T, Cho IC, et al. Estabilishment of a microsatellite marker set for individual, pork brand and product origin identification in pigs. J Anim Sci Technol. 2009;51:201-6.

10. Cheng HH, Crittenden LB. Microsatellite markers for genetic mapping in the chicken. Poult Sci. 1994;73:539-46.

11. Crooijmans RP, Groen AF, Van Kampen AJ, Van der Beek S, Van der Poel JJ, Groenen MA. Microsatellite polymorphism in commercial broiler and layer lines estimated using pooled blood samples. Poult Sci. 1996;75:904-9.

12. Abasht B, Dekkers JCM, Lamont SJ. Review of quantitative trait loci identified in the chicken. Poult Sci. 2006;85:2079-96.

13. Food and Agriculture Organization of the United Nations. Molecular genetic characterization of animal genetic resources. Rome: FAO Animal production and Health Guidelines; 2011. No. 9: 84-85.

14. Seo DW, Hoque MR, Choi NR, Sultana H, Park HB, Heo KN, et al. Discrimination of Korean native chicken lines using fifteen selected Microsatellite markers. Asian-Aust J Anim Sci. 2013;26:316-22.

15. Marshall T, Slate J, Kruuk L, Pemberton J. Statistical confidence for likelihood-based paternity inference in natural populations. Mol Ecol. 1998;7:639-55

16. Ayres KL, Overall AD. api-calc 1.0: a computer program for calculating the average probability of identity allowing for substructure, inbreeding and the presence of close relatives. Mol Ecol Notes. 2004;4:315-8.

17. Pritchard JK, Stephens M, Donnelly P. Inference of population structure using multilocus genotype data. Genetics. 2000;155:945-59.

18. Jombart $\mathrm{T}$. adegenet: a $\mathrm{R}$ package for the multivariate analysis of genetic markers. Bioinformatics. 2008;24:1403-5.

19. Jombart T, Devillard S, Balloux F. Discriminant analysis of principal components: a new method for the analysis of genetically structured populations. BMC Genetics. 2010;11:94.

20. Jombart T, Collins C, Solymos P, Ahmed I, Calboli F, Cori A. Package 'adegenet'. ftp://mint.c3sl.ufpr.br/CRAN/web/packages/adegenet/adegenet pdf. Accessed June 7, 2014

21. Nei M, Tajima F, Tateno Y. Accuracy of estimated phylogenetic trees from molecular data. J Mol Evol. 1983;19:153-70.

22. Liu K, Muse SV. PowerMarker: an integrated analysis environment for genetic marker analysis. Bioinformatics. 2005;21:2128-9.

23. Paradis E, Claude J, Strimmer K. APE: analyses of phylogenetics and evolution in R language. Bioinformatics. 2004;20:289-90.

24. Huson DH, Bryant D. Application of phylogenetic networks in evolutionary studies. Mol Biol Evol. 2006;23:254-67.

25. Suh S, Cho CY, Kim JH, Choi SB, Kim YS, Kim H, et al. Analysis of genetic characteristics and probability of individual discrimination in Korean indigenous chicken brands by microsatellite marker. J Anim Sci Technol. 2013;55:185-94.

26. Botstein D, White RL, Skolnik M, Davis RW. Construction of a genetic linkage map in man using restriction fragment length polymorphisms. Am J Hum Genet. 1980;32:314-31.

27. Evanno G, Regnaut S, Goudet J. Detecting the number of clusters of individuals using the software STRUCTURE: a simulation study. Mol Ecol. 2005; 14:2611-20.

\section{Submit your next manuscript to BioMed Central and take full advantage of:}

- Convenient online submission

- Thorough peer review

- No space constraints or color figure charges

- Immediate publication on acceptance

- Inclusion in PubMed, CAS, Scopus and Google Scholar

- Research which is freely available for redistribution 